\title{
El Instituto de Estudios \\ Internacionales de la Universidad de Chile
}

C L $\propto U$ D to VÉ L I z es el Director del Instituto de Estudios Internacionales de in Universidad de Chile. Fntre 1962 y 1965 ocupó el puesto de Senior Research Fellow en el Royal Institute of International Affairs, de Londr... $D$ utor de una Historia de la Marina Mercanle de Chile (Santiago, 1961), y dirigió la preparación de Obslacles to Change in Latin America (Lon. dres, 1965), $y$ The Politics of Conformity in Latin dmerica, (Londres, 196i).

Aun cuando durante el siglo $x \mathrm{x}$ y las primeras décadas del actual, las nacio. nes de América Latina desarrollaron una activa y compleja diplomacia y se preocuparon preferentemente de sus respectivos problemas internacionales, ninguna alcanzó suficiente polerio o importancia como para influir de modo significativo en los asuntos políticos mundiales. No por esto es menos interesante Ia experiencia acumulada durante ese periodo: un siglo de ejercicio notablemente libre de una política internacional independiente dejó huellas profundas tanto en la idiosincrasia de los grupos dirizentes como en la tradición histórica de estas naciones. La experiencia politica internacional de Américā Latina es un microcosmos que incluye varias guerras internacionales, arbitrajes, pactos y alianzas, diplomacia secreta, intentonas expansionistas y complicados sistemas de balanzas y equilibrios de fuerzs militares.

Todo esto contribuyó a formar una robusta pero sofisticada tradición aisiacionista que dependia - en la práctica- no sólo de la relativa debilidad de cada una de estas naciones, sino también del conrencimiento de que esta parte del mundo era realmente independiente de los intereses, rencores y amistades que determinaban el curso de la politica mundial. Este convencimiento se afirmó decididamente durante los difíciles años de 1914 a 1918 cuando las principales naciones de América Latina mantuvieron una actitud de estricta neutralidad.

Es posible que la crisis de 1929 haya marcado el principio del fin de este aislacionismo. El fenómeno cconómico que remeció las bases de la estructura institucional de América Latina tuvo origenes lejanos que escaparon a toda ingerencia nuestra. Después de 1929, ni cl más testarudo pudo permitirse 
cerrar los ojos a lo que ocurría fucra del continente: partidos populistas, agrupaciones de ideario importado, frentes populares y movimientos reformistas, todos empezaron a mirar hacia afuera, esta vez no en busca de modelos para imitar, sino genuinamente interesados en el rumbo de la politica mundial y empeñados en lograr voz y voto en su determinación. Incluso, es importante anotarlo, el primer centro para el estudio de los asuntos internacionales de que se tenga memoria en América Latina, de efimera existencia, fue fundado por un grupo de chilenos hacia las medianias de la década de 1930.

El estallido de la Segunda Guerra Mundial puso fin a todo esto al congelar la actividad diplomática y dividir al mundo en mitades que se excluían absolutamente.

La Guerra Fria prolongó la forzada inmovilidad politica internacional privando a los países de América Latina de la posibilidad real de examinar críticamente sus respectivas posiciones en el concierto mundial y tratar de establecer las bases para una política exterior independiente $y$ con proyecciones que alcanzaran fuera del ámbito del continente. Durante este periodo las actitudes, planteamientus e intereses de los contendores eran perfectamente predecibles e incompatibles; inciuso hubo quienes consideraron que no era prudente ni permisible acercarse a estudiar estos problemas desde un punto de vista estrictamente académico.

Esta situación ha cambiado fundamentalmente: entre los primeros sintomas del conflicto chino-soviético y la ahora famosa conferencia de prensa del Presidente De Gaulle en enero de 3963, se inició el fin de la Guerra Fría, generalizándose a ambos lados de la difunta 'Cortina de Hierro' una clara tendencia policentrista que indudablemente ha significado un retorno de la fluidez a los asuntos políticos internacionales. Los dos gigantes termonucleares se encuentran paradójicamente inmovilizados, tanto por la tremenda responsabilidad que implica la posesión de tan grande poder destructivo, como por la orfandad ideológica y la falta de dirección en que quedaron sumidos cuando, simultáneamente con disminuir sus recelos mutuos se debilitó también el imperativo primordial de la elaborada política de Guerra Fría. Hoy incluso es posible sostener que el desafio politico planteado por China es tanto o más importante para la Unión Soviética como la rivalidad de los Estados Unidos; asimismo, no seria extraño que los Estados Unidos se sinticran más desafiados por la posición politica de Francia que por la de Rusia. Ya no es posible continuar analizando las relaciones entre las naciones en función de la existencia de dos bloques monoliticos de poder que abrazan ideologias absolutamente incompatibles. Por el contrario, las demandas prácticas de la política internacional han atenuado considerablemente el celo doctrinario de las dos grandes potencias y han dado a su gestión politica externa un acentuado tono empirico. Esto ha acompañado al retroceso de la marca alta de la Guera Fria que ha dejado al descubierto las cumbres elevadas de los intereses nacionales, sumergidas durante las dos decadas en que la proliteración de 
Closdio l'ili: / El Instituto de Estudios Insernacionalos de b Universidad de Chile arreglos supranacionales defensivos y ofensivos, hicieron crecr que se encon. traba en marcha una decidida tendencia intermacionalista.

Hoy dia parece probable que el nucvo complejo politico nundial será construjdo precisamente sobre la apreciación, más o menos bien entendida, de estos intereses nacionales.

Esta extraordinaria y paradójica restauración de la libertad de maniobra luego de tan pro.ongada inmovilidad representa una oportunidad de desarrollar una politica exterior dinśmica e influyente en el concierto mundial que los paises principales de América Latina parecen dispuestos a aprovechar. Tal situación traerá consigo responsabilidades de importancia creciente $y$, entendiéndolo-así, el Rector de la Universidad de Chile y el Hon. Consejo Universitario convinieron en que la Universidad debía interesarse en forma eficiente y sistemática en la investigación de los problemas de las relaciones internacionales, $y$ en la divulgación de datos y conceptos serios sobre estos asuntos.

Para cumplir estos propósitos se creó el Instituto de Estudios Internacio nales en 1966 y aparece ahora el primer número de la revista trimestral. Estu: dios Internacionales.

Es, imposible ignorar el hecho de que la creación de este Instituto coincide con un momento interesantísimo y pleno de posibilidades en la historia politica de América Latina. Estạ situación favorable acarrea también riesgos importantes; abundarán las presiones para que el nuevo centro de estudios se incline en esta o aquella dirección, apoye tales o cuales políticas o exprese indirectamente su aprobación por determinadas tendencias en el panorama político mundial. Por esto es de gran importancia el hacer notar que el Instituto, además de participar de la tradicional independencià que ha caracterizado las actividades de la casa universitaria principal de la nación, ha sido planeado esencialmente como un centro interdisciplinario para trabajar en estrechia colaboración con las cuatro facultades universitarias que se preocupan de adelantar los estudios en disciplinas afines al campo general de las relaciones internacionales y la historia contemporánea. Además, dentro de la estructura administrativa de la Universidad de Chile, el Instituto depende directamente de la Rectoria y del Hon. Consejo Universitario, asegurándose asi la autonomía que otorga seriçdad, desinterés y rigor académico a sus trabajos.

El nuevo centro tiene parentesco enorgullecedor con los principales institutos del mundo en que se estudian estos problemas; en su formación ha tenido un papel importantísimo el apoyo sostenido del Roy'al Institule of Intcrnatio: nal Affairs, de Londres, y del Centre d'Eludes de Politique Etrangdre, de Paris. Sin embargo, es útil:aclarar, ninguno de estos dos famosos centros ha servido como modelo absoluto para la creación del nuevo Instituto. Tanto la organización interna como las relaciones con el Gobiemo y con los sectores principales de la vida nacional han sido planteadas sobre una base estrictamente 


\section{ESTUDIOS IXTER X ACIONALES}

pragnática y lo mís acorde posible con ha necesidades acadimicas y las posibilidades que enciern nuestro conglomerado nacional y latinonmericano; se ha tomado la experiencia valiosa acumulada durante varias déadas en Europa y se ha modificado de acuerdo con las clemandas funcionales que plantea nuestro medio.

Por ejemplo, aun cuando el Instituto es esencialmente un centro de investigación, también cumplirí una función docente. al nivel postgraduado a diferencia del Rojal Instilute of Inlernational Affairs- sencillamente porque tal función es necesaria en nuestro país y continente. Esto no quiere decir que el Instituto quiera transformarse en centro de adiestramiento para quienes se interesen por la carrera diplomítica. lin primer lugar, ya existe un prestigioso plantel -la Academia Andrćs Bello del Mrinisterio de Relaciones Exteriores de Chile- que llena este cometicio en forma perfectamente adccuada; en segundo lugar, los cursos del Instituto no scguirán programas regulares ni pretenderán de modo alguno cubrir el campo de las relaciones internacionales en forma sistemática o exhaustiva. Por ejemplo, en 1967, algunos de los cursos versarán sobre la situación politica en el sureste de Asia, Africa al Sur del Sahara y Europa, mientras que otras tratarán de explicar las complejidades de la situación económica internacional de América Latina. En 1968, se ofrecerán dos nuevos ciclos; uno estará dedicado a examinar aspectos de los procesos de integración europeo y latinoamericano y el otro consistirá en varios cursos sobre la política exterior de los principales países de América Latina. Además de estos cursos, el Instituto ha buscado otros medios para poder desempeñar una función docente efectiva y de alto nivel, que contribuya al enriquecimiento del pensamiento sobre estos problemas. Conjuntamente con el Centre d'Etudes de Politique Etrangère, de Paris, por ejemplo, se está'organizando un seminario especializado que se reunirá en Arica en enero de 1968 , a analizar en detalle la experiencia europea en materia de integración política y económica, con el caso de América Latina en mente. Sc contará en esta oportunidad con la participación de algunos de los principales arquitectos de] Mercado Común Europeo y muchos de los que hoy día en América Latina buscan el camino de la integración.

Asimismo, funcionarán en forma regular en el Instituto un seminario sobre problemas" contemporáneos de América Latina y un Grupo de Estudios sobre política internacional.

Para adelantar todos estos proyectos y cumplir cficientemente las tareas programadas, el Instituto ha buscado la cooperación de centros dedicados al estudio de disciplinas afines en América Latina y el resto dol mundo; así se están estableciendo estrechas relaciones de consulta y colaboración -además de los institutos ya mencionados- con el Instilut des Sciences Economique Appliquees, el Inslitul du Developpemenl Economique et Social, ambos de Paris; el Instituto Otto Sulrr, de la Universidad de Berlin; el Instituto Arnold Bergstraesser, de la Universidad de Freiburg; el Instituto para la Integración 


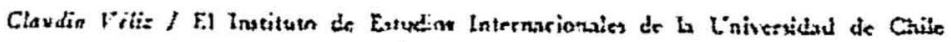
de Ameria Latina, de Bucnos Aires; el Instituto Brasileño de Asuntos Infernacionalcs; cl Colegio de Mexico, y la London School of Economics and Polilical Scicnce.

Desde sus comienzos, e! Instituto de Estudios Internacionales de la Unifersidad de Chile tiene relaciones funcionales con los principales centros en que sc analizan estos problemas. Esto le da acceso a los mis elevados niveles del pensamiento mundial sobre las relaciones económicas y políticas entre las naciones y resulta en una corriente continua de renovación y crítica que asegura el rigor académico sobre ci que se funclamentarán sus trabajos de investigación.

El primer centro mundial para el estudio de los problemas internacionales contemporáncos fuc el Rojal Institute of International Affairs, que nació a la sombra de las negociaciones de Versalles en 1919 y tenía como indisimulado propósito el contribuir a la mantención de la paz mundial. El nuevo Instituto de Estudios Internacionales no tiene propósitos normativos comparables. Estimando que la satisfacción de la curiosidad inteligente del hombre no necesita justificación, pretende declicar sus mejores esfuerzos al análisis de. los procesos politicos y económicos contemporáneos que más honda marca dejan en la historia internacional de nuestro tiempo.

Así como es encomiable que una universidad que se respete dedique tiempo y esfuerzo a la clasificación exhaustiva de aves y batracios, asimismo, es lícito y deseable que invierta recursos en el adelanto de la investigación cientifica de las causas y consecuencias del sistema apartheid en Ia República Sudafricana; del conflicto de Vietnam; Ia emergencia en Aden, o el proceso de integración latinoamericano. $Y$ todo esto no por un falso empirismo que sugiera que tales estudios conduzcan a políticas exteriores más acertadas o cosas por el estilo, sino sencillamente porque éstos son conflictos, procesos y situaciones que envuelven el destino, la vida y la muerte de miles y millones de seres humanos y las actividades de nuestros congéneres deben ser siempre, y esencialmente, objeto de la más elevada y digna preocupacićn académica. 